\title{
Rac1 mediates phorbol 12-myristate 13-acetate-induced migration of glioblastoma cells via paxillin
}

\author{
NAOKO NOMURA ${ }^{1}$, MOTOHIRO NOMURA $^{2}$, NOBUHISA MIZUKI $^{1}$ and JUN-ICHIRO HAMADA ${ }^{3}$ \\ ${ }^{1}$ Department of Ophthalmology and Visual Science, Yokohama City University School of Medicine, Yokohama 236-0004; \\ ${ }^{2}$ Department of Neurosurgery, Yokohama Sakae Kyosai Hospital, Yokohama 247-8581; ${ }^{3}$ Department of Neurosurgery, \\ Kanazawa University Graduate School of Medical Science, Kanazawa 920-8641, Japan
}

Received March 26, 2008; Accepted May 12, 2008

DOI: 10.3892/or_00000063

\begin{abstract}
Previously, we reported that phorbol 12-myristate 13-acetate (PMA)-activated protein kinase C (PKC) induced Rac1 activation in A172 glioblastoma cells. In this study, we investigated the mechanism of PMA-activated PKC-induced migration of glioblastoma cells by focusing on Rac1. PMAinduced formation of lamellipodia and focal complexes following migration were blocked by inhibiting Rac1 with small interfering RNA (siRNA), implicating Rac1 in PMAinduced glioblastoma cell migration. PMA-activated PKC induced phosphorylation of c-jun N-terminal kinase (JNK), one of the downstream effectors of Rac1. Immunohistochemical analysis showed that phosphorylated JNK was translocated to paxillin-containing focal complexes upon PMA stimulation and that Rac1 siRNA blocked these phenomena. These results suggest that phosphorylated JNK functions in cell migration and that JNK phosphorylation and translocation are mediated by Rac1. Furthermore, inhibition of Rac 1 reduced phosphorylation of paxillin, a focal adhesion component and a downstream effector of JNK, at serine 178 $\left(\mathrm{Ser}^{178}\right)$. Paxillin phosphorylation at this site has been shown to be involved in cell migration. Immunohistochemical analysis detected phosphorylation of paxillin $\left(\operatorname{Ser}^{178}\right)$ in focal complexes upon PMA stimulation that was blocked by Rac1 siRNA. SP600125, a JNK inhibitor, also blocked PMAinduced phosphorylation of paxillin and aggregation of phosphorylated paxillin $\left(\operatorname{Ser}^{178}\right)$ in focal complexes. In conclusion, paxillin is a critical downstream effector of Rac1 that may be involved in PMA-stimulated migration presumably by modulating the integrity of focal complex formation.
\end{abstract}

Correspondence to: Dr Motohiro Nomura, Department of Neurosurgery, Yokohama Sakae Kyosai Hospital, 132 Katsura-cho, Sakae-ku, Yokohama 247-8581, Japan

E-mail: nomura413jp@yahoo.co.jp

Key words: glioblastoma, migration, phorbol 12-myristate 13acetate, protein kinase $\mathrm{C}$, Rac1, c-jun N-terminal kinase, paxillin

\section{Introduction}

Glioblastoma is a highly aggressive tumor for which no adequate treatment has yet been developed. The malignant progression of astrocytomas is thought to occur via multistep processes that result in the degradation of cellular growth control.

One of these processes has been reported to involve overexpression or hyperactivity of protein kinase C (PKC) (1). PKC is a family of phospholipid-dependent serine/ threonine kinases. Like other kinases, PKCs have been found to be involved in intracellular signal transduction pathways that regulate cell growth, differentiation and apoptosis. In malignant gliomas, rapid proliferation rates have been functionally linked to inherently high levels of PKC and subsequent in excessive activation of PKC-mediated pathways (2).

To investigate the molecular mechanism underlying PKC-induced cell migration, phorbol 12-myristate 13-acetate (PMA) was used to activate PKC. PMA-activated PKC has been implicated in rearrangement of the cytoskeleton and migration of U251MG glioblastoma cells $(3,4)$.

Previously, we reported that PMA-activated PKC induced Rac1 activation in the leading edge of migrating A172 glioblastoma cells (5). Rac1 is a member of the Rho GTPase family of intracellular signal transducers. Like small GTPases of the Ras family, Rho GTPases act as molecular switches and cycle between an active (GTP-bound) and inactive (GDPbound) status. Previous studies demonstrated that Rac1 is one of the key signaling components controlling the organization of the actin cytoskeleton. It was shown that Rac1 is activated in the frontal region of migrating cells (6) and thought to induce lamellipodia by stimulating actin polymerization. In glioblastomas, siRNA-mediated depletion of Rac1 strongly inhibits invasive behavior of SNB19 and U87MG glioblastoma cells (7). Rac1 has a number of downstream effectors including mitogen-activated protein kinases (MAPK) such as c-jun N-terminal kinase (JNK) and p38MAPK (8-11).

JNK has been reported to have essential roles in inflammation, differentiation, and apoptosis. Moreover, there are accumulating data indicating an involvement of JNK in cell migration. It has been suggested that JNK regulates migration by phosphorylation of paxillin at serine 178 
$\left(\operatorname{Ser}^{178}\right)$ in fish keratocytes and rat bladder tumor endothelial cells (12).

It was reported that Rac1 might control the turnover of focal adhesion complexes (13), but the mechanism is not yet known. In this study, we analyzed the molecular mechanisms by which PMA-activated PKC can induce glioblastoma cell migration by focusing on the effect of Rac1. We demonstrated that paxillin is a critical downstream component of Rac1 that may participate in PKC-induced migration by modulating the integrity of focal adhesions.

\section{Materials and methods}

Reagents and antibodies. PMA and bisindolylmaleimide I (BIS) were purchased from Sigma-Aldrich Corporation (St. Louis, MO). SP600125, a JNK inhibitor, was purchased from Calbiochem (San Diego, CA). PMA, BIS and SP600125 were prepared as $10 \mathrm{mM}$ stock solutions in dimethyl sulfoxide (DMSO). The primary antibodies used were mouse antipaxillin monoclonal antibody (BD Transduction Laboratories, Franklin Lakes, NJ); rabbit anti-phospho-paxillin $\left(\mathrm{Ser}^{178}\right)$ polyclonal antibody (Chemicon, Temecula, CA); rabbit antiSAPK/JNK polyclonal antibody (Cell Signaling Technology, Beverly, MA); rabbit anti-phospho-SAPK/JNK (Thr183/ Tyr185) polyclonal antibody (Cell Signaling Technology); and mouse anti-vinculin monoclonal antibody (Sigma-Aldrich Corp.).

Cell lines. A172 glioblastoma cells were obtained from American Type Culture Collection. The cells were maintained in Dulbecco's modified Eagle medium (DMEM) (Bio Whittaker, Rockland, ME) supplemented with $10 \%$ fetal bovine serum (FBS; Atlanta Biologicals, Norcross, GA), $100 \mathrm{U} / \mathrm{ml}$ penicillin, $100 \mu \mathrm{g} / \mathrm{ml}$ streptomycin and $2 \mathrm{mM}$ glutamine (Gibco BRL, Grand Island, NY) in a humidified atmosphere containing $5 \% \mathrm{CO}_{2}$ and $95 \%$ air at $37^{\circ} \mathrm{C}$. Cells were passaged every 3-4 days to ensure logarithmic growth.

Stimulation of PKC by a specific inducer. PMA was used to activate PKC. The glioblastoma cells were incubated in serum-free medium for $24 \mathrm{~h}$. PMA was then added to the culture medium and the cells were cultured for 0-120 min.

Inhibition of kinase activity by specific inhibitors. BIS and SP600125 were used for the inhibition of PKC and JNK activity, respectively. Initially, glioblastoma cells were cultured in serum-free medium for $24 \mathrm{~h}$. Then BIS or SP600125 was added to the culture medium to a final concentration of 5 or $10 \mu \mathrm{M} 30$ min prior to the addition of PMA. Cells treated with inhibitors were incubated with PMA for $60 \mathrm{~min}$ and subjected to further analysis.

Migration assay. Cells were seeded in 12-well culture dishes (BD Sciences) and cultured until they became subconfluent. The cells were then incubated with serum-free culture medium for $24 \mathrm{~h}$, scraped with a $200-\mu 1$ micro-pipet tip, and washed with PBS. The cells were incubated with serum-free culture medium for an additional $16 \mathrm{~h}$ with PMA or vehicle $(5,14)$. When specific inhibitors were used, an appropriate concentration of each inhibitor was added to the culture medium
30 min before PMA treatment. After $16 \mathrm{~h}$ of incubation, the cells were photographed, and the migrated area was measured using NIH Image software.

Transfection of siRNA into glioblastoma cells. siRNA for Rac1 was purchased from Dharmacon (Tokyo, Japan). For control experiments, control siRNA-A (Santa Cruz, Santa Cruz, CA) was used. Reagents for transfection (Lipofectamine 2000 and Opti-MEM I reduced serum medium) were obtained from Invitrogen (Carlsbad, CA). Transfection of siRNA into the glioblastoma cells was done according to the manufacturer's instructions. After a 48-h incubation of the cells with siRNA, the cells were cultured for an additional $24 \mathrm{~h}$ with serum-free medium. The transfected cells were incubated with PMA or vehicle for $60 \mathrm{~min}$ and then subjected to Western blot analysis or fluorescence immunohistochemistry.

Western blot analysis. Total cells were harvested from each culture condition at the appropriate time intervals and washed with ice-cold PBS. The total protein was then extracted using lysis buffer containing $1 \%$ Triton X-100, $150 \mathrm{mM} \mathrm{NaCl}, 5 \mathrm{mM}$ EDTA, $50 \mathrm{mM}$ sodium fluoride, $1 \mathrm{mM}$ sodium orthovanadate, $5 \mu \mathrm{g} / \mathrm{ml}$ phenylmethylsulfonyl fluoride, $2 \mu \mathrm{g} / \mathrm{ml}$ aprotinin, $5 \mu \mathrm{g} / \mathrm{ml}$ leupeptin and $2 \mu \mathrm{g} / \mathrm{ml}$ pepstatin. The samples were centrifuged at $15000 \mathrm{rpm}$ for $30 \mathrm{~min}$ at $4^{\circ} \mathrm{C}$, and the extracts were stored at $-80^{\circ} \mathrm{C}$ until use. Protein concentration was determined using the BCA assay (Pierce, Rockford, IL). For Western blot analysis, equal amounts of protein $(20-30 \mu \mathrm{g})$ were electrophoresed on an SDS-PAGE gel, transferred to a nitrocellulose membrane (Trans-Blot Transfer Medium 0.45 micron, Bio-Rad, Hercules, CA) and stained with Ponceau S (Sigma-Aldrich Corp.). After confirmation of protein transfer, proteins were detected with specific antibodies. All primary antibodies were used at a dilution of $1: 1000$. Actin protein was detected as a control with a mouse anti-human B-actin monoclonal antibody (Chemicon) used at a 1:10000 dilution. Sheep anti-mouse IgG or donkey antirabbit IgG horseradish peroxidase-linked secondary antibodies (Amersham, Piscateway, NJ) at a 1:4000 dilution were used as secondary antibodies. Protein detection was performed using SuperSignal West Femto Maximum Sensitivity Substrate (Pierce) and signals were visualized using Hyperfilm ECL (Amersham).

Fluorescence immunohistochemistry. Cells were plated in 8well chamber slides and incubated overnight. After attachment of the cells to the slides, the cells were incubated with serumfree culture medium for $24 \mathrm{~h}$. After a 30-min pre-treatment with specific inhibitors or vehicle, $100 \mathrm{nM}$ PMA was added to the culture medium without serum and the cells were incubated for $60 \mathrm{~min}$. Then, the cells were fixed with $3.7 \%$ paraformaldehyde for $20 \mathrm{~min}$ and washed with PBS three times. The cells were permeabilized with $0.2 \%$ Triton X-100 for $5 \mathrm{~min}$ and washed with PBS three times. After blocking with $2 \% \mathrm{BSA}$, the cells were incubated at room temperature with specific primary antibodies at a dilution of $1: 100$. Then the cells were washed with PBS three times and were incubated at room temperature with fluorescence-labeled secondary antibodies at a 1:200 dilution. After washing with 
A

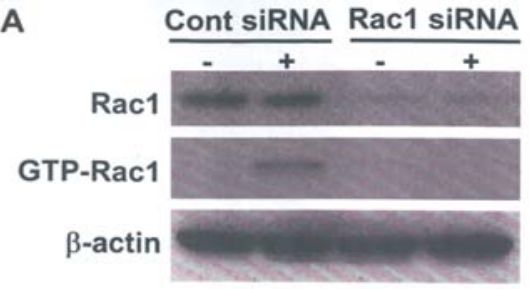

B
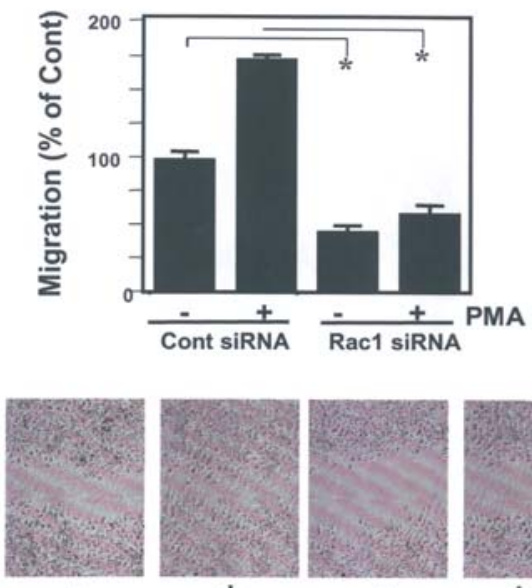

Cont siRNA

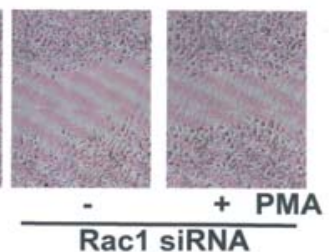

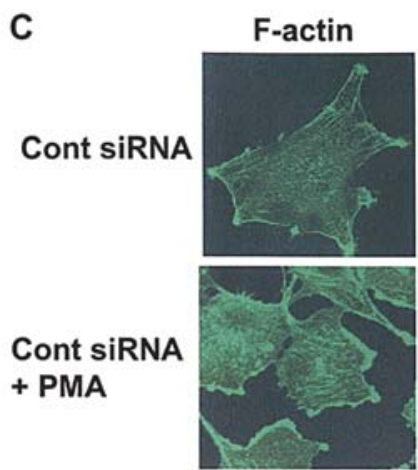
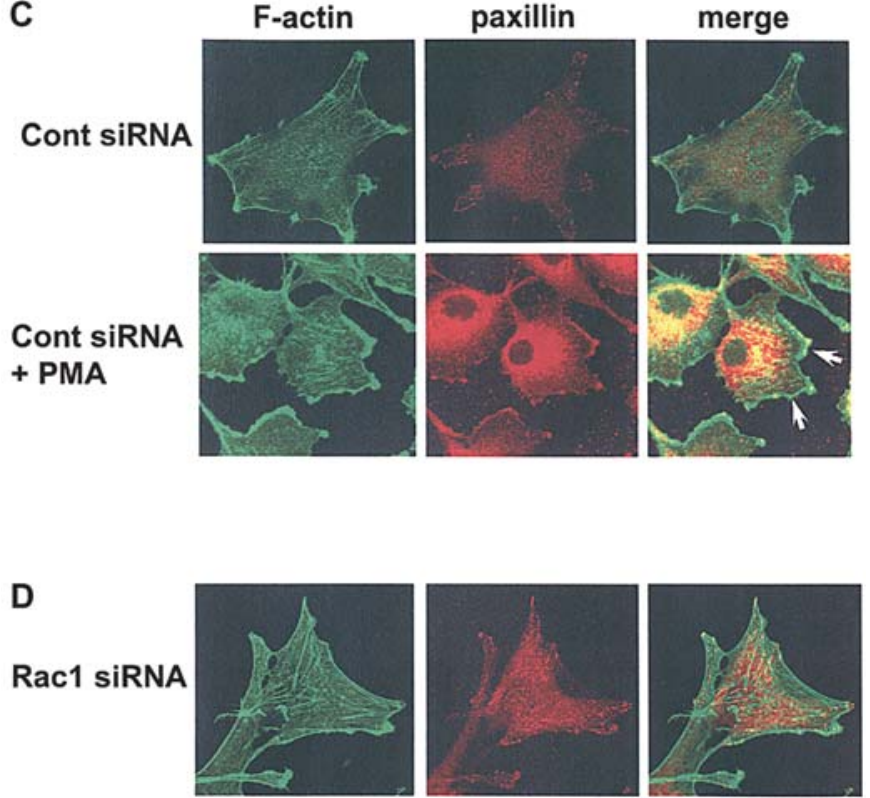

Rac1 siRNA

+ PMA
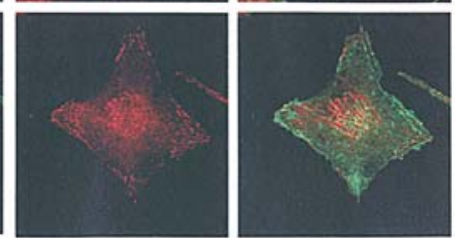

Figure 1. Effect of Rac1 inhibition on glioblastoma cells. (A) Cells transfected with Rac1 or control siRNA were stimulated with PMA or vehicle for 60 min. Expression and activation of Rac1 were examined by Western blot analysis. The amount of ß-actin was determined by reprobing the membrane with an antiB-actin antibody after stripping. (B) Rac1 siRNA-transfected cells were incubated without serum for $24 \mathrm{~h}$ and the cells were wounded. After $16 \mathrm{~h}$ of stimulation with PMA, migration was evaluated. Results from three independent experiments are presented. Migration is expressed as the percentage of unstimulated cells. " p $<0.05$. (C, D) The transfected cells were treated with PMA or vehicle for 60 min after serum-starvation. After fixation, the cells were double-labeled for F-actin and paxillin. F-actin and paxillin are labeled green and red, respectively. Yellow fluorescence shows colocalization of F-actin and paxillin. Arrows indicate the lamellipodia.

PBS, the samples were mounted. Alexa fluor 488 phalloidin (Invitrogen) was used for detection of F-actin, which appeared green under a fluorescence microscope. The secondary antibodies used were Alexa fluor 594 goat anti-rabbit antibody and Alexa fluor 594 goat anti-mouse antibody (Invitrogen). Both secondary antibodies appeared red under a fluorescence microscope.

Assay for Racl activation. The Rac-GTP pull-down assay was performed using a Rac1 activation assay kit (Upstate Biotechnology, Charlottesville, VA) according to the manufacturer's instructions. Briefly, the cell lysates were incubated with PAK-1 PBD agarose to bind Rac1-GTP. Proteins bound to PAK-1 PBD were separated by SDS-PAGE, transferred to a nitrocellulose membrane, and probed with Rac1 antibody.

Statistical analysis. All experiments were performed at least three times. The data are expressed as the mean \pm standard error of the mean (SEM). Probability (P) values were calculated using Student's t-test. P-values $<0.05$ were considered significant.

\section{Results}

Knockdown of endogenous Racl by siRNA blocked PMAinduced glioblastoma cell migration. We have recently shown that stimulation of PKC by PMA activates Rac1 through the Src/Cas/Crk signaling pathway in A172 glioblastoma cells (14). We have also demonstrated that PMA-activated PKC induces glioblastoma cell migration (5).

To investigate the underlying mechanism of Rac1-mediated cell migration in PMA-stimulated cells, we transfected A172 glioblastoma cells with Rac1 siRNA. The efficiency of the siRNA treatment in reducing protein levels was confirmed by Western blot analysis (Fig. 1A). Rac1 expression was decreased to $18 \%$ of the control. Further, to detect Rac1 activation, a PAK PBD GST pull-down assay was performed to detect GTP-bound Rac1. Consistent with our previous report (14), Rac1 was activated 5 min after PMA stimulation and this activation was abolished by Rac1 siRNA transfection (Fig. 1A).

We then performed a migration assay. As shown in Fig. 1B, PMA-induced cell migration was blocked by Rac1 siRNA. The data indicate that Rac1 is critical for PMA-induced glioblastoma migration.

It is known that at the leading edge of migrating cells, actin-polymerized lamellipodia are formed, and focal complexes are formed under the lamellipodia (15). To analyze the effect of Rac1 on changes in the actin cytoskeleton and focal complex formation, immunohistochemical study was performed. To detect F-actin, phalloidin was used. For detection of focal adhesions, paxillin, one of the most 

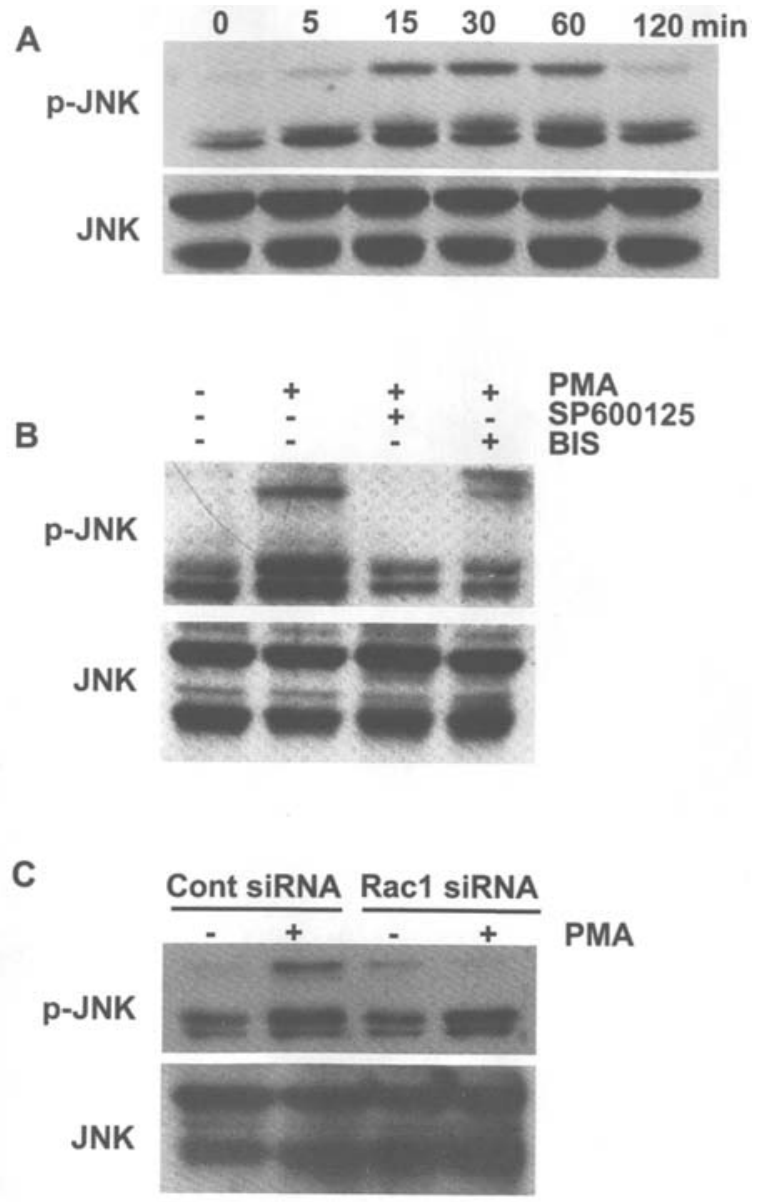

Figure 2. Effect of PMA and inhibitors on expression and phosphorylation of JNK. (A) Cells were treated with $100 \mathrm{nM}$ PMA for the indicated times. (B) BIS or SP600125 was added to serum-starved cells 30 min prior to PMA stimulation. Cells were then treated with $100 \mathrm{nM}$ PMA for $60 \mathrm{~min}$. (C) Cells transfected with Rac1 or control siRNA were stimulated with PMA or vehicle for $60 \mathrm{~min}$. Expression and phosphorylation of JNK were examined by Western blot analysis.

prominent residents of focal adhesions, was stained. As shown in Fig. 1C and D, formation of F-actin and focal adhesions in Rac1 siRNA-transfected cells was almost identical to the control. As previously reported $(5,14)$, PMA treatment induced lamellipodia formation and dot-like focal complexes under lamellipodia (Fig. 1C, lower panel). On the other hand, in Rac1 siRNA-transfected cells, lamellipodia formation was inhibited and large focal adhesions were observed in the cytosol (Fig. 1D, lower panel). These data indicate that PMA-induced formation of lamellipodia and focal complexes were dependent on Rac1.

PMA-induced phosphorylation of JNK and translocation to focal complexes were dependent on Rac1. Rac1 has a large number of downstream effectors and so it is important to define which downstream pathway is affected. JNK is a Rac1 downstream effector (16) that could potentially affect Rac1induced cell migration.

We next addressed whether Rac1-induced focal complex formation was associated with increased JNK-signaling in glioblastoma cells. We first tested whether JNK was activated by PMA-activated PKC. As shown in Fig. 2A, phosphorylated JNK (p-JNK) increased after 15 min of PMA treatment and
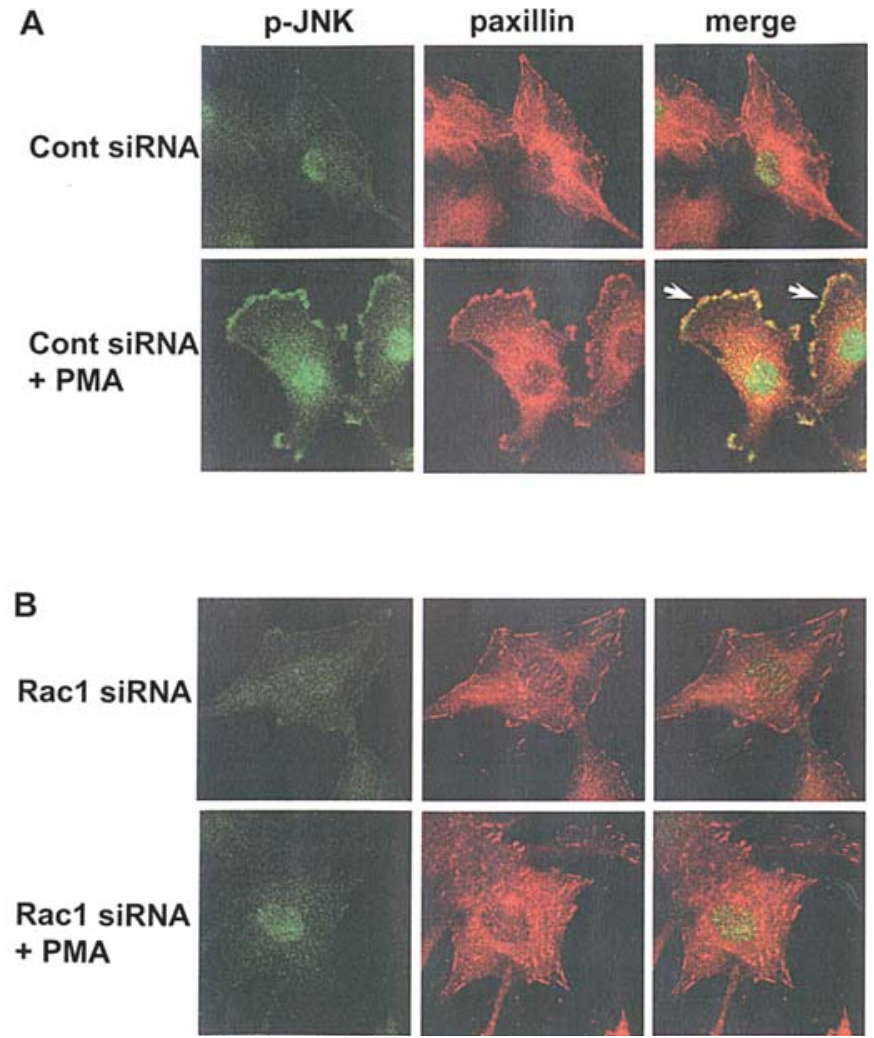

Figure 3. Distribution of p-JNK and paxillin. Cells were transfected with control (A) or Rac1 (B) siRNA. After serum starvation, cells were treated with PMA or vehicle for $60 \mathrm{~min}$. After fixation, the cells were doublelabeled for p-JNK and paxillin. p-JNK and paxillin are labeled green and red, respectively. Yellow fluorescence shows colocalization of p-JNK and paxillin. Arrows indicate the lamellipodia.

this phenomenon was largely abolished by the PKC inhibitor BIS (Fig. 2B). These results indicate that PMA induced JNK phosphorylation in a PKC-dependent manner.

Then, to test the possibility that phosphorylation of JNK was mediated by Rac1, we transfected the cells with Rac1 siRNA. As shown in Fig. 2C, knockdown of Rac1 by siRNA resulted in significant inhibition of PMA-induced phosphorylation of JNK with no change in the basal JNK level. These results showed that PMA-induced phosphorylation of JNK was mediated via Rac1.

Next, to test whether JNK has a role in focal complex formation during PMA-induced migration, immunohistochemical analysis was used to detect $\mathrm{p}$-JNK and paxillin. As shown in Fig. 3A, p-JNK showed slight staining in the nuclei of control cells. After PMA treatment, p-JNK was detected both in nuclei and in paxillin-containing focal complexes. BIS pretreatment blocked these phenomena, showing that PMA-induced translocation of $\mathrm{p}$-JNK to focal complexes is dependent on PKC activation (data not shown).

Then, to analyze whether PMA-induced translocation of p-JNK to paxillin-containing focal complexes is dependent on Rac1, cells transfected with Rac1 siRNA were doublestained for p-JNK and paxillin. In Rac1-suppressed cells, both PMA-induced focal complex formation at the cell periphery and translocation of $\mathrm{p}$-JNK to paxillin-containing focal complexes were inhibited, and large focal adhesions were detected in the cytosol. These data suggest that activation of JNK at paxillin-containing focal complexes is a part of the 
A

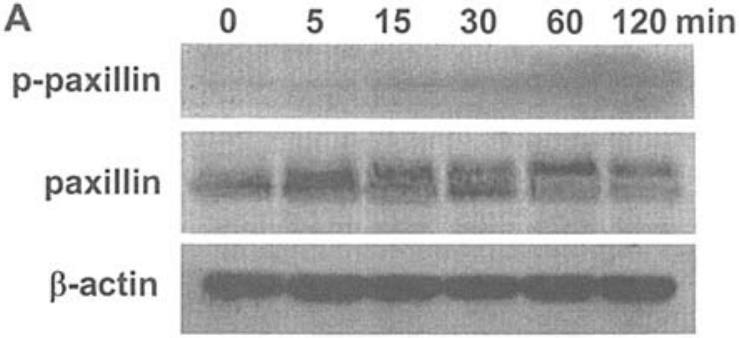

B
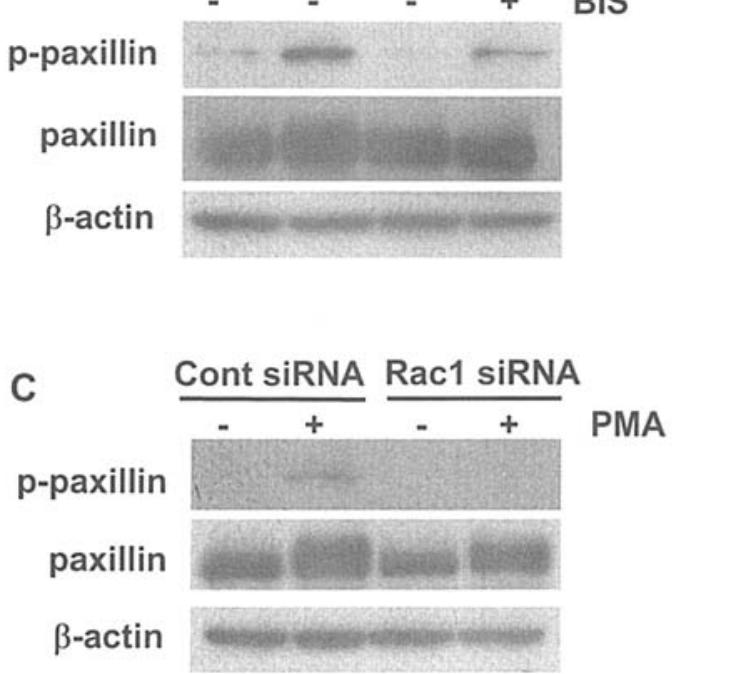

Figure 4. Effect of PMA and inhibitors on expression and phosphorylation of paxillin. (A) Cells were treated with $100 \mathrm{nM}$ PMA for the indicated times. (B) BIS or SP600125 was added to serum-starved cells 30 min prior to PMA stimulation. Cells were then treated with $100 \mathrm{nM}$ PMA for $60 \mathrm{~min}$. (C) Cells transfected with siRNA were incubated with PMA or vehicle for $60 \mathrm{~min}$. Expression and phosphorylation of paxillin were examined by Western blot analysis.

mechanism by which migration signals originating from PMA-activated PKC are transduced by Rac1.

PMA phosphorylated paxillin at Ser ${ }^{178}$ via Rac1. JNK efficiently phosphorylates paxillin at $\operatorname{Ser}^{178}$ and promotes following cell migration (12). The JNK inhibitor SP600125 blocked phosphorylation of Ser ${ }^{178}$ of paxillin and decreased migratory activity of EGF-stimulated human breast cancer cells (12) or PDGF-stimulated foreskin fibroblasts (17). On the basis of these previous studies, together with our finding that JNK is a downstream effector of Rac1 in PMA-stimulated glioblastoma cells (Fig. 2C), we investigated whether PMAactivated $\mathrm{PKC}$ could promote paxillin phosphorylation at $\operatorname{Ser}^{178}$ and if so whether this process is involved in Rac1 activation.

As shown in Fig. 4A, we found that $\mathrm{Ser}^{178}$ of paxillin was phosphorylated after 15 min of PMA treatment with the effect persisting for at least $120 \mathrm{~min}$. Paxillin phosphorylation was largely inhibited by BIS, indicating that paxillin phosphorylation by PMA was dependent on PKC (Fig. 4B). Furthermore, pretreatment with SP600125 blocked PMAinduced phosphorylation of paxillin (Fig. 4B), indicating that PMA-induced phosphorylation of paxillin was mediated by JNK activation.
To confirm that the signaling pathway is located downstream of Rac1, we next examined whether Rac1 is required for PMA-activated PKC-induced phosphorylation of paxillin at $\mathrm{Ser}^{178}$. As expected, transfection of Rac1 siRNA blocked phosphorylation of paxillin (Fig. 4C) in addition to JNK (Fig. 2C). These results indicate that PMA/PKC induced phosphorylation of paxillin at $\operatorname{Ser}^{178}$ through activation of Rac1 and JNK.

p-paxillin $\left(\mathrm{Ser}^{178}\right)$ was detected at the leading edge of PMA/ $P K C$-induced migrating cells, and its expression was dependent on Racl. To examine the role of paxillin phosphorylation at focal adhesions during cell migration, we doublestained the cells for F-actin and p-paxillin $\left(\operatorname{Ser}^{178}\right)$. Fig. 5A (upper panel) shows that p-paxillin $\left(\operatorname{Ser}^{178}\right)$ could be faintly observed in non-migrating cells. On the other hand, PMA treatment induced lamellipodia formation and aggregation of p-paxillin $\left(\operatorname{Ser}^{178}\right)$ at the leading edges of the lamellipodia (Fig. 5A, lower panel). These results are consistent with previous findings that p-paxillin $\left(\operatorname{Ser}^{178}\right)$ is a critical component for cell migration (12).

To confirm that p-paxillin $\left(\operatorname{Ser}^{178}\right)$ was co-localized with focal complexes, the expression of p-paxillin $\left(\operatorname{Ser}^{178}\right)$ and another focal adhesion protein, vinculin, were analyzed by immunohistochemistry. Upon PMA stimulation, dot-like focal complexes were found at the leading edge, and the colocalized paxillin was confirmed to be phosphorylated at $\operatorname{Ser}^{178}$ (Fig. 5B). These phenomena were inhibited by pretreatment with BIS, indicating that expression of p-paxillin $\left(\right.$ Ser $\left.{ }^{178}\right)$ was mediated by PKC activation (data not shown).

To strengthen our finding that PMA-induced paxillin at the leading edge (Fig. 1C) is phosphorylated, double-staining for paxillin and p-paxillin (Ser ${ }^{178}$ ) was performed. Fig. 5C showed that paxillin appeared at the leading edge of cells containing p-paxillin $\left(\operatorname{Ser}^{178}\right)$.

Then, to reveal the role of Rac 1 in focal complex regulation, double-staining for p-paxillin $\left(\mathrm{Ser}^{178}\right)$ and paxillin was done in the cells transfected with Rac1 siRNA. As shown in Fig. 5D, the paxillin observed in Rac1 siRNA-transfected cells was not colocalized with a p-paxillin $\left(\operatorname{Ser}^{178}\right)$ signal. These findings further indicate that Rac1 was involved in the phosphorylation of paxillin at $\operatorname{Ser}^{178}$.

Furthermore, we performed immunohistochemical analyses to examine the formation of focal complexes under JNK inhibition. As shown in Fig. 5E, SP600125 treatment blocked PMA-induced focal complex formation and induced the appearance of large focal adhesions. These large focal adhesions did not contain p-paxillin (Ser $\left.{ }^{178}\right)$. Cytoskeletal changes were similarly observed in cells transfected with Rac1 siRNA (Fig. 5D). These results implicate PMAactivated PKC-induced Rac1 and JNK activation in both paxillin phosphorylation and expression at focal complexes.

\section{Discussion}

Although involvement of PKC in glioblastoma cell migration has been reported (2), the mechanisms have not been completely revealed. We have recently shown that the stimulation of PKC by PMA activates Rac1 through the Src/ Cas/Crk signaling pathway in A172 glioblastoma cells (14). 
A

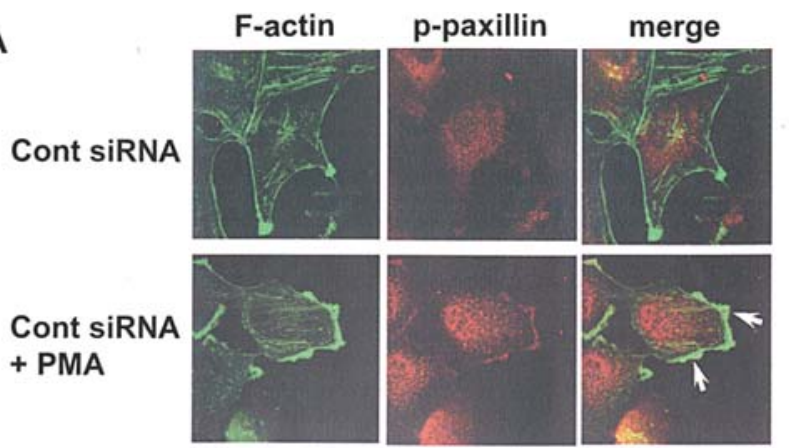

B

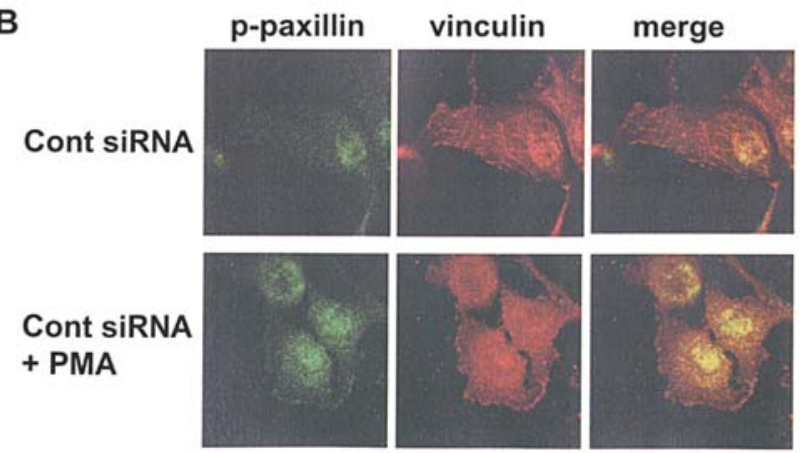

Figure 5. Distribution of F-actin, vinculin, paxillin and p-paxillin. Cells were treated with PMA or vehicle for $60 \mathrm{~min}$ after serum starvation. After fixation, the cells were double-labeled for F-actin and p-paxillin (A) or for p-paxillin and vinculin (B). (A) F-actin and p-paxillin are labeled green and red, respectively. Yellow fluorescence shows the region of colocalization. Arrows indicate the lamellipodia. (B) p-paxillin and vinculin are labeled green and red, respectively. (C, D) Cells transfected with Rac1 or control siRNA were treated with PMA or vehicle for $60 \mathrm{~min}$. (E) Cells incubated in serum-free medium for $24 \mathrm{~h}$ were pre-treated with SP600125 for $30 \mathrm{~min}$ and then treated with PMA. After fixation, the cells were double-labeled for p-paxillin and paxillin. p-paxillin and paxillin are labeled green and red, respectively.

Accumulating data show that the Rac1/JNK (18) and JNK/ paxillin (12) pathways are both involved in cell migration. However, a definitive causal relationship between Rac1 activity and adhesion contact components such as paxillin has not been shown.

In this study, we examined the role of Rac1 in PMAactivated PKC-induced migration of glioblastoma cells by focusing on focal adhesion component, paxillin. We showed that Rac1 was involved in glioblastoma cell migration (Fig. 1B), and that JNK is a downstream effector of the PMA/PKC/Rac1 pathway. PMA-activated PKC induced phosphorylation of JNK, and p-JNK was detected in paxillincontaining focal complexes in glioblastoma cells (Fig. 3). Further, the paxillin in the focal complexes was phosphorylated at $\mathrm{Ser}^{178}$ (Fig. 5). These data indicate that JNK activation at focal complexes might play a role in paxillin phosphorylation. Western blot analysis showed that inhibition of JNK by SP600125 blocked PMA-induced phosphorylation of paxillin at $\mathrm{Ser}^{178}$, suggesting that JNK activation might modulate paxillin phosphorylation. Our observations are consistent with previous reports that JNK phosphorylated paxillin at $\mathrm{Ser}^{178}$, and that p-paxillin was directly associated with metastasis of hepatocellular carcinoma (19).

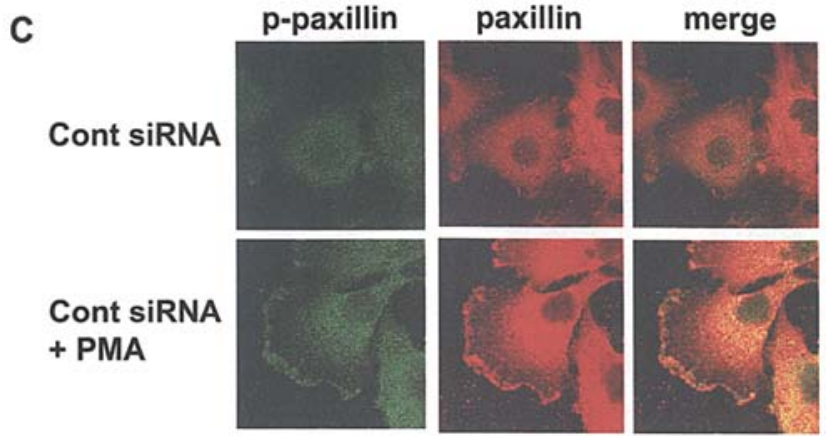

D
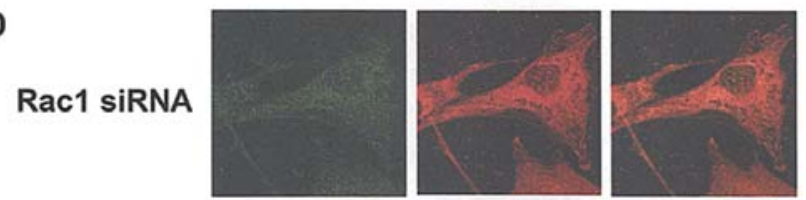

\section{Rac1 siRNA + PMA}
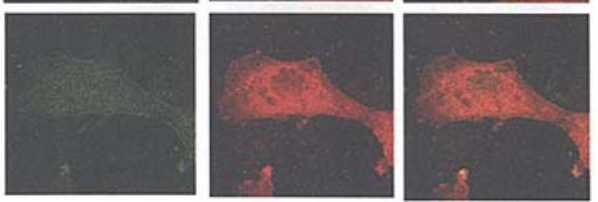

E

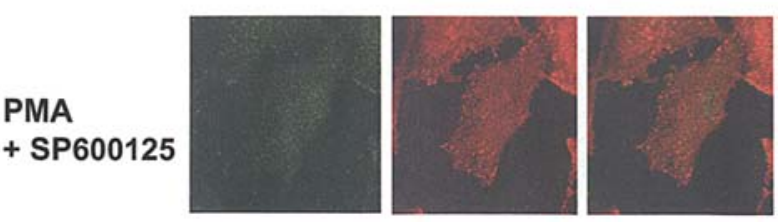

PMA-induced phosphorylation of paxillin at $\operatorname{Ser}^{178}$ was also blocked by Rac1 siRNA (Fig. 4C). Further, Rac1suppressed cells failed to induce the formation of focal complexes, and large focal adhesions in the cytosol did not contain p-paxillin (Ser ${ }^{178}$ ). These large focal adhesions might inhibit membrane protrusion.

To generalize our findings, LN18 and Hs683 glioblastoma cells were also used for experiments. We found that PMAinduced cell migration was blocked by Rac1 siRNA in both LN18 and Hs683 cells (data not shown). These data indicate that cell migration induced by PMA/Rac1 is not cell linedependent.

We showed that Rac1 regulates glioblastoma cell migration through paxillin phosphorylation; however the question of whether Rac1 targets other cytoskeletal components during cell migration still remains. Rac1 has a large number of downstream effectors, including kinases such as p38MAPK (20). We previously reported that PMA/PKC-induced actin polymerization and lamellipodia formation were dependent on the p38MAPK/Hsp27 pathway (5). It is possible that cell migration mediated by Rac1 is dependent on the JNK/paxillin pathway for focal complex formation and also other pathways such as p38MAPK/Hsp27 for lamellipodia formation.

In summary, we demonstrated that Rac 1 is a critical component in PMA-induced glioblastoma cell migration. Rac1-mediated glioblastoma cell migration may occur via activation of JNK and phosphorylation of paxillin (Ser $\left.{ }^{178}\right)$. Although the involvement of p-paxillin $\left(\operatorname{Ser}^{178}\right)$ in cell migration has been reported, the mechanism is not clear. 
Further investigation of the role of p-paxillin $\left(\operatorname{Ser}^{178}\right)$ in cell migration is necessary.

\section{Acknowledgements}

This study was supported in part by Grants-in-Aid for Encouragement of Scientists (Grant No. 17924036 to N.N., 18924037 and 19924026 to M.N.) from Japan Society for the Promotion of Science.

\section{References}

1. Sharif TR and Sharif M: Overexpression of protein kinase C epsilon in astroglial brain tumor derived cell lines and primary tumor samples. Int J Oncol 15: 237-243, 1999.

2. Da Rocha AB, Mans DR, Regner A and Schwartsmann G: Targeting protein kinase $\mathrm{C}$ : new therapeutic opportunities against high-grade malignant gliomas? Oncologist 7: 17-33, 2002.

3. Besson A, Davy A, Robbins SM and Yong VW: Differential activation of ERKs to focal adhesions by PKC epsilon is required for PMA-induced adhesion and migration of human glioma cells. Oncogene 20: 7398-7407, 2001.

4. Besson A, Wilson TL and Yong VW: The anchoring protein RACK1 links protein kinase Cepsilon to integrin beta chains. Requirements for adhesion and motility. J Biol Chem 277: 22073-22084, 2002.

5. Nomura N, Nomura M, Sugiyama K and Hamada J: Phorbol 12-myristate 13-acetate (PMA)-induced migration of glioblastoma cells is mediated via p38MAPK/Hsp27 pathway. Biochem Pharmacol 74: 690-701, 2007.

6. Del Pozo MA, Price LS, Alderson NB, Ren XD and Schwartz MA: Adhesion to the extracellular matrix regulates the coupling of the small GTPase Rac to its effector PAK. EMBO J 19: 2008-2014, 2000.

7. Chan AY, Coniglio SJ, Chuang YY, et al: Roles of the Rac1 and Rac3 GTPases in human tumor cell invasion. Oncogene 24 : 7821-7829, 2005

8. Bagrodia S, Derijard B, Davis RJ and Cerione RA: Cdc42 and PAK-mediated signaling leads to Jun kinase and p38 mitogenactivated protein kinase activation. J Biol Chem 270: 27995-27998, 1995 .
9. Coso OA, Chiariello M, Yu JC, et al: The small GTP-binding proteins Rac1 and Cdc42 regulate the activity of the JNK/SAPK signaling pathway. Cell 81: 1137-1146, 1995.

10. Minden A, Lin A, Claret FX, Abo A and Karin M: Selective activation of the JNK signaling cascade and c-Jun transcriptional activity by the small GTPases Rac and Cdc42Hs. Cell 81: 1147-1157, 1995.

11. Zhang S, Han J, Sells MA, et al: Rho family GTPases regulate p38 mitogen-activated protein kinase through the downstream mediator Pak1. J Biol Chem 270: 23934-23936, 1995.

12. Huang C, Rajfur Z, Borchers C, Schaller MD and Jacobson K: JNK phosphorylates paxillin and regulates cell migration. Nature 424: 219-223, 2003

13. Nobes CD and Hall A: Rho GTPases control polarity, protrusion and adhesion during cell movement. J Cell Biol 144: 1235-1244, 1999.

14. Nomura N, Nomura M, Sugiyama K and Hamada J: Src regulates phorbol 12-myristate 13 -acetate-activated $\mathrm{PKC}$-induced migration via Cas/Crk/Rac1 signaling pathway in glioblastoma cells. Int J Mol Med 20: 511-519, 2007.

15. Yamaguchi $H$ and Condeelis J: Regulation of the actin cytoskeleton in cancer cell migration and invasion. Biochim Biophys Acta 1773: 642-652, 2007.

16. Hall A: Rho GTPases and the control of cell behaviour. Biochem Soc Trans 33: 891-895, 2005.

17. Amagasaki K, Kaneto H, Heldin $\mathrm{CH}$ and Lennartsson J: c-Jun N-terminal kinase is necessary for platelet-derived growth factor-mediated chemotaxis in primary fibroblasts. J Biol Chem 281: 22173-22179, 2006.

18. Juliano RL, Reddig P, Alahari S, Edin M, Howe A and Aplin A: Integrin regulation of cell signalling and motility. Biochem Soc Trans 32: 443-446, 2003.

19. Ching YP, Leong VY, Lee MF, Xu HT, Jin DY and Ng IO: P21-activated protein kinase is overexpressed in hepatocellular carcinoma and enhances cancer metastasis involving c-Jun $\mathrm{NH} 2$-terminal kinase activation and paxillin phosphorylation. Cancer Res 67: 3601-3608, 2007.

20. Shin I, Kim S, Song H, Kim HR and Moon A: H-Ras-specific activation of Rac-MKK3/6-p38 pathway: its critical role in invasion and migration of breast epithelial cells. J Biol Chem 280: 14675-14683, 2005. 